\title{
A methodology for the design of robust rollover prevention controllers for automotive vehicles: Part 1-Differential braking
}

\author{
Selim Solmaz*, Martin Corless and Robert Shorten
}

\begin{abstract}
In this paper we apply recent results from robust control to the problem of rollover prevention in automotive vehicles. Specifically, we exploit the results of Pancake, Corless and Brockman, which provide controllers to robustly guarantee that the peak values of the performance outputs of an uncertain system do not exceed certain values. We introduce a new measure of performance for rollover prevention, the Load Transfer Ratio $L T R_{d}$, and design differentialbraking-based rollover controllers to keep the value of this quantity below a certain level; we also obtain controllers which yield robustness to variations in vehicle speed. We present numerical simulations to demonstrate the efficacy of our controllers.
\end{abstract}

\section{INTRODUCTION}

It is well known that vehicles with a high center of gravity such as vans, pickups, and the highly popular SUVs (Sport Utility Vehicles) are more prone to rollover accidents. According to the 2004 data [1], light trucks (pickups, vans and SUV's) were involved in nearly $70 \%$ of all the rollover accidents in the USA, with SUV's alone responsible for almost $35 \%$ of this total. The fact that the composition of the current automotive fleet in the U.S. consists of nearly $36 \%$ pickups, vans and SUV's [2], along with the recent increase in the popularity of SUV's worldwide, makes rollover an important safety problem.

There are two distinct types of vehicle rollover: tripped and un-tripped. A tripped rollover commonly occurs when a vehicle slides sideways and digs its tires into soft soil or strikes an object such as a curb or guardrail. Driver induced un-tripped rollover can occur during typical driving situations and poses a real threat for top-heavy vehicles. Examples are excessive speed during cornering, obstacle avoidance and severe lane change maneuvers, where rollover occurs as a direct result of the lateral wheel forces induced during these maneuvers. In recent years, rollover has been the subject of intensive research, especially by the major automobile manufacturers; see, for example, [3], [4]. That research is geared towards the development of rollover prediction schemes and occupant protection devices. It is however, possible to prevent such a rollover incident by monitoring the car dynamics and applying proper control effort ahead of time. Therefore there is a need to develop driver assistance technologies which would be transparent to the driver during normal driving conditions, while acting in emergency situations to recover handling of the vehicle until the driver recovers control of the vehicle [5].

We present in this paper a robust rollover prevention controller design methodology based on differential braking. The proposed control design is an application of recent results on the design of control systems which guarantee that the peak values of the performance outputs of a plant do not exceed certain thresholds when subject to bounded disturbance inputs [6], [7]. The main

S. Solmaz (selim.solmaz@nuim.ie) and R. Shorten (robert.shorten@nuim.ie) are with the Hamilton Institute, National University of Ireland-Maynooth, Ireland. M. Corless (corlessepurdue.edu) is with the School of Aeronautics \& Astronautics, Purdue University, West Lafayette, IN, USA.

${ }^{*}$ Corresponding author. Phone:+353 17086100 , Fax: +353 17086269 selected performance output for the rollover problem is the Load Transfer Ratio $L T R_{d}$. This measure of performance is related to tire lift-off and it can be considered as an early indicator of impending vehicle rollover. We also include the braking force as a performance output to take into account limitations on the maximum braking force. The aim of our control strategy is to maximize the magnitude of the allowable disturbance inputs which do not drive the performance outputs outside their prespecified limits; in this case the disturbance input is the driver steering input. We also want to guarantee robustness with respect to the parameter uncertainty that arises from changing vehicle speed. We indicate how our design can be extended to account for other sources of uncertainty such as unknown vehicle center of gravity and tire stiffness parameters.

\section{RELATED WORK}

Rollover prevention is a topical area of research in the automotive industry (see, for example, http://www.safercar.gov/Rollover for a good introduction to the problem) and several studies have recently been published. Relevant publications include that of Palkovics et al. [8], where they proposed the ROP (Roll-Over Prevention) system for use in commercial trucks making use of the wheel slip difference on the two sides of the axles to estimate the tire lift-off prior to rollover. Wielenga [9] suggested the ARB (Anti Roll Braking) system utilizing braking of the individual front wheel outside the turn or the full front axle instead of the full braking action. The suggested control system is based on lateral acceleration thresholds and/or tire lift-off sensors in the form of simple contact switches. Chen et al. [10] suggested using an estimated TTR (Time To Rollover) metric as an early indicator for the rollover threat. When TTR is less than a certain preset threshold value for the particular vehicle under interest, they utilized differential braking to prevent rollover. Ackermann et al. and Odenthal et al. [11], [12] proposed a robust active steering controller, as well as a combination of active steering and emergency braking controllers. They utilized a continuous-time active steering controller based on roll rate measurement. They also suggested the use of a static Load Transfer Ratio $\left(L T R_{S}\right)$ which is based on lateral acceleration measurement; this was utilized as a criterion to activate the emergency steering and braking controllers.

\section{VeHICLE MODELLING AND $L T R_{d}$}

In this section we introduce the model that we use for controller design. We also define the rollover detection criterion $L T R_{d}$ and present the assumptions on the sensors and actuators used in the design.

\section{A. Vehicle Model}

We use a linearized vehicle model for control design. Specifically, we consider the well known single-track model with a roll degree of freedom. In this model the steering angle $\delta$, the roll angle $\phi$ and the vehicle sideslip angle $\beta$ are all assumed to be small. We 

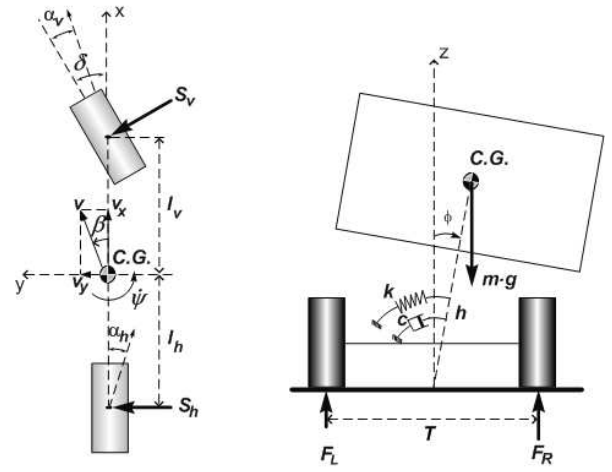

Fig. 1. Linear bicycle model with roll degree of freedom.

further assume that all the vehicle mass is sprung, which implies insignificant unsprung mass. The lateral forces on the front and rear tires, denoted by $S_{v}$ and $S_{h}$, respectively, are represented as linear functions of the tire slip angles $\alpha_{v}$ and $\alpha_{h}$, that is, $S_{v}=C_{v} \alpha_{v}$ and $S_{h}=C_{h} \alpha_{h}$, where $C_{v}$ and $C_{h}$ are the front and rear tire stiffness parameters, respectively. In order to simplify the model description, we further define the following auxiliary variables

$$
\begin{aligned}
& \sigma \triangleq C_{v}+C_{h}, \\
& \rho \triangleq C_{h} l_{h}-C_{v} l_{v}, \\
& \kappa \triangleq C_{v} l_{v}^{2}+C_{h} l_{h}^{2},
\end{aligned}
$$

where the lengths $l_{v}$ and $l_{h}$ are defined in Figure 1. For simplicity, it is assumed that, relative to the unsprung mass, the sprung mass rolls about a horizontal roll axis which is along the centerline of the unsprung mass and at ground level. Using the parallel axis theorem of mechanics, $J_{x_{e q}}$, the moment of inertia of the vehicle about the assumed roll axis, is given by

$$
J_{x_{e q}}=J_{x x}+m h^{2},
$$

where $h$ is the distance between the vehicle center of gravity (CG) and the assumed roll axis and $J_{x x}$ is the moment of inertia of the vehicle about the roll axis through the CG. Introducing the state $x=\left[\begin{array}{llll}\beta & \dot{\psi} & \dot{\phi} & \phi\end{array}\right]^{T}$, where $\dot{\psi}$ is the yaw rate of the unsprung mass, the motion of this model can be described by

$$
\dot{x}=A x+B_{\delta} \delta+B_{u} u
$$

where

$$
\begin{aligned}
A & =\left[\begin{array}{cccc}
-\frac{\sigma J_{x e q}}{m J_{x x} v} & \frac{\rho J_{x e q}}{m J_{x x} v^{2}}-1 & -\frac{h c}{J_{x x} v} & \frac{h(m g h-k)}{J_{x x} v} \\
\frac{\rho}{J_{z z}} & -\frac{\kappa}{J_{z z} v} & 0 & 0 \\
-\frac{h \sigma}{J_{x x}} & \frac{h \rho}{J_{x x} v} & -\frac{c}{J_{x x}} & \frac{m g h-k}{J_{x x}} \\
0 & 0 & 1 & 0
\end{array}\right], \\
B_{\delta} & =\left[\begin{array}{llll}
\frac{C_{v} J_{x e q}}{m J_{x x} v} & \frac{C_{v} l_{v}}{J_{z z}} & \frac{h C_{v}}{J_{x x}} & 0
\end{array}\right]^{T}, \\
B_{u} & =\left[\begin{array}{llll}
0 & -\frac{T}{2 J_{z z}} & 0 & 0
\end{array}\right]^{T}
\end{aligned}
$$

and $u$ represents the differential braking force on the wheels; it is positive if braking is on the right wheels and negative if braking is on the left wheels. Differential braking force as the control input is depicted in Figure 2 below. Note that we can brake either front, rear or both of the wheels on each side of the vehicle depending on the maneuver and $u$ is the total effective braking force acting on either side as illustrated in the figure. Further definitions for all

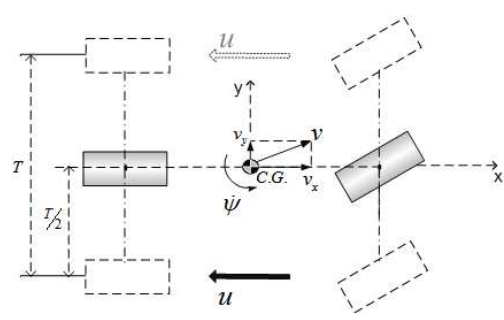

Fig. 2. Differential braking force as control input.

TABLE I

MODEL PARAMETERS AND THEIR DEFINITIONS

\begin{tabular}{cll}
\hline Parameter & Description & Unit \\
\hline$m$ & vehicle mass & {$[\mathrm{kg}]$} \\
$v$ & vehicle speed & {$[\mathrm{m} / \mathrm{s}]$} \\
$\delta$ & steering angle & {$[\mathrm{rad}]$} \\
$J_{x x}$ & roll moment of inertia at CG & {$\left[\mathrm{kg} \cdot \mathrm{m}^{2}\right]$} \\
$J_{z z}$ & yaw moment of inertia at CG & {$\left[\mathrm{kg} \cdot \mathrm{m}^{2}\right]$} \\
$l_{v}$ & longitudinal CG position w.r.t. front axle & {$[\mathrm{m}]$} \\
$l_{h}$ & longitudinal CG position w.r.t. rear axle & {$[\mathrm{m}]$} \\
$T$ & vehicle track width & {$[\mathrm{m}]$} \\
$h$ & distance of CG from roll axis & {$[\mathrm{m}]$} \\
$c$ & suspension damping coefficient & {$[\mathrm{N} \cdot \mathrm{m} \cdot \mathrm{s} / \mathrm{rad}]$} \\
$k$ & suspension spring stiffness & {$[\mathrm{N} \cdot \mathrm{m} / \mathrm{rad}]$} \\
$C_{v}$ & linear tire stiffness for front tire & {$[\mathrm{N} / \mathrm{rad}]$} \\
$C_{h}$ & linear tire stiffness for rear tire & {$[\mathrm{N} / \mathrm{rad}]$} \\
\hline
\end{tabular}

the parameters in (4) are given in Table I. See [13] for a detailed derivation of this vehicle model.

In order to model the change in the vehicle speed $v$ as a simple function of the braking force, we assume that the longitudinal wheel forces generated by the engine counteract the rolling resistance and the aerodynamic drag at all times. Under this assumption, the vehicle speed is approximately governed by

$$
\dot{v}=-\frac{|u|}{m} \text {. }
$$

\section{B. The Load Transfer Ratio, LTR}

Traditionally, as discussed in the related work section, some estimate of the vehicle load transfer ratio (LTR) has been used as a basis for the design of rollover prevention systems. The quantity LTR [12], [14] can be simply defined as the load (i.e., vertical force) difference between the left and right wheels of the vehicle, normalized by the total load. In other words

$$
L T R=\frac{\text { Load on Right Tires-Load on Left Tires }}{\text { Total Load }}
$$

Clearly, $L T R$ varies within $[-1,1]$, and for a perfectly symmetric car that is driving straight, it is zero. The extrema are reached in the case of a wheel lift-off of one side of the vehicle, in which case LTR becomes 1 or -1 depending on the side that lifts off. If roll dynamics are ignored, it is easily shown [12] that the corresponding LTR (which we denote by $L T R_{S}$ ) is approximated by

$$
L T R_{s} \triangleq \frac{2 a_{y} h}{g T}
$$

where $a_{y}$ is the lateral acceleration of the CG.

A contribution of this paper is to note that rollover estimation based upon (7) is not sufficient to detect the transient phase of rollover (due to the fact that it is derived ignoring roll dynamics.) Consequently, we obtain an expression for LTR which does not ignore roll dynamics. We denote this by $L T R_{d}$. In order to derive 
$L T R_{d}$ we write a torque balance equation. Recall that we assumed the unsprung mass is insignificant and the main body of the vehicle rolls about an axis along the centerline of the unsprung mass at the ground level. We can write a torque balance for the unsprung mass about the assumed roll axis in terms of the suspension torques and the vertical wheel forces as follows:

$$
-F_{R} \frac{T}{2}+F_{L} \frac{T}{2}-k \phi-c \dot{\phi}=0 .
$$

Now substituting the definition of LTR from (6) and approximating the total load by the vehicle weight, yields the following expression for $L T R_{d}$ :

$$
L T R_{d}=-\frac{2(c \dot{\phi}+k \phi)}{m g T} .
$$

In terms of the state $x, L T R_{d}$ can be described by

$$
L T R_{d}=C_{1} x,
$$

where

$$
C_{1}=\left[\begin{array}{llll}
0 & 0 & -\frac{2 c}{m g T} & -\frac{2 k}{m g T}
\end{array}\right] .
$$

\section{Actuators, Sensors and Parameters}

We are interested in control design based on differential braking. Active braking actuators are already available in many modern production cars that are equipped with systems such as ABS (Antilock Braking System) and EBS (Electronic Brake System) or similar systems, which are capable of selectively braking each of the wheels. The fact that control designs using these actuators can be commissioned without much financial overhead makes them the preferred actuator candidates in the literature.

We also assume full state feedback information for the design of the controllers and that all the model parameters are known. This is an unrealistic assumption; however, our control design is easily extended to account for uncertainty in these parameters. As a side note, although we assumed all the vehicle model parameters to be known, it is possible to estimate some of these that are fixed (but unknown) using the sensor information available for the control design suggested here; this however is outside the scope of this work [15].

\section{State Feedback Controllers for Robust Disturbance ATtEnUATION}

We are interested in designing a controller to prevent rollover that is robust with respect to parameter uncertainty. Our starting point is in results obtained by Pancake, Corless and Brockman for uncertain systems of the form

$$
\begin{aligned}
\dot{x} & =A(\theta) x+B(\theta) w+B_{u}(\theta) u \\
z_{i} & =C_{i}(\theta) x+D_{i u}(\theta) u, \quad i=1, \ldots, r
\end{aligned}
$$

where $x(t) \in \mathbb{R}^{n}$ is the state at time $t \in[0, \infty), w(t) \in \mathbb{R}^{m}$ is a bounded disturbance input, $u(t) \in \mathbb{R}^{m_{u}}$ is the control input, and $z_{i}(t) \in \mathbb{R}^{p_{i}}, i=1,2, \ldots, r$ are performance outputs. All the uncertainty and nonlinearities in the system are captured in the parameter vector $\theta$ which can depend on $t, x, w$ and $u$. We wish to synthesize a stabilizing controller which prevents the peak values of the performance outputs exceeding certain values. In other words, we want to design a feedback controller, which guarantees a bounded performance output given a bounded uncertain disturbance, that is, $\|w(t)\| \leq w_{\max }$. We consider linear state feedback controllers of the form

$$
u=K x,
$$

where $K$ is a constant state feedback gain matrix. This results in a closed loop system described by

$$
\begin{aligned}
\dot{x} & =\left[A(\theta)+B_{u}(\theta) K\right] x+B(\theta) w \\
z_{i} & =\left[C_{i}(\theta)+D_{i u}(\theta) K\right] x, \quad i=1, \ldots, r .
\end{aligned}
$$

We require the following assumptions.

Assumption 1: There are matrices

$$
A_{j}, B_{j}, B_{u j}, \quad j=1, \ldots, N
$$

so that for each $\theta$, the matrix $\left[A(\theta) B(\theta) B_{u}(\theta)\right]$ can be written as a convex combination of $\left[A_{1} B_{1} B_{u 1}\right], \ldots,\left[A_{N} B_{N} B_{u N}\right]$.

Assumption 2: For each $i=i, \ldots, r$, there are matrices

$$
C_{i k}, D_{i u k}, \quad k=1, \ldots, M_{i}
$$

so that for each $\theta$, the matrix $\left[C_{i}(\theta) D_{i u}(\theta)\right]$ can be written as a convex combination of $\left[C_{i 1} D_{i u 1}\right], \ldots,\left[C_{i M_{i}} D_{i u M_{i}}\right]$.

Remark 1: Suppose that each of the matrices $A(\theta), B(\theta), B_{u}(\theta)$ depends in a multi-affine fashion on the components of an $\bar{L}$-vector $\theta$ and each element of $\theta$ is bounded; specifically,

$$
\underline{\theta}_{l} \leq \theta_{l} \leq \bar{\theta}_{l} \quad \text { for } \quad l=1, \ldots, \bar{L} .
$$

Then, for all $\theta$, the matrix $\left[A(\theta) B(\theta) B_{u}(\theta)\right]$ can be expressed as a convex combination of the $2^{\bar{L}}$ vertex matrices corresponding to the extreme values of the components of $\theta$, that is, $\theta_{l}=$ $\underline{\theta}_{l}$ or $\bar{\theta}_{l}$ for $l=1, \ldots, \bar{L}$.

We have now the following result which is useful for control design.

Theorem 1: Consider a nonlinear/uncertain system described by (12)-(13) and satisfying Assumptions 1 and 2. Suppose that there exist matrices $S=S^{T}>0$ and $L$ along with scalars $\alpha_{1}, \ldots, \alpha_{N}>0$ and $\gamma_{1}, \ldots, \gamma_{r} \geq 0$ such that the following matrix inequalities hold:

$$
\left[\begin{array}{cc}
A_{j} S+B_{u j} L+S A_{j}^{T}+L^{T} B_{u j}^{T}+\alpha_{j} S & B_{j} \\
B_{j}^{T} & -\alpha_{j} I
\end{array}\right] \leq 0,
$$

for $j=1, \ldots, N$ and

$$
\left[\begin{array}{cc}
-S & S C_{i k}^{T}+L^{T} D_{i u k}^{T} \\
C_{i k} S+D_{i u k} L & -\gamma_{i}^{2} I
\end{array}\right] \leq 0
$$

for $i=1, \ldots, r$ and $k=1, \ldots, M_{i}$. Then the controller

$$
u=K x \quad \text { with } \quad K=L S^{-1}
$$

results in a closed loop nonlinear/uncertain system which has the following properties.

(a) The undisturbed system $(w=0)$ is globally exponentially stable, that is, all state trajectories decay exponentially.

(b) If the disturbance input is bounded, that is, $\|w(t)\| \leq w_{\max }$ for all $t$ then, for zero initial state, the performance outputs $z_{1}, \ldots, z_{r}$ of the closed loop system are bounded and satisfy

$$
\left\|z_{i}(t)\right\| \leq \gamma_{i} w_{\max }
$$

The scalars $\gamma_{1}, \ldots, \gamma_{r}$ are called performance levels and can be regarded as measures of the ability of the closed loop system to attenuate the effect of the disturbance input on the performance outputs; a smaller $\gamma_{i}$ means better performance in the sense of increased attenuation. For a proof of the theorem, see [7]. 


\section{Rollover Prevention CONTROllers}

Here we use the results of the previous section to obtain rollover prevention controllers using differential braking as the control input. We consider the driver's steering wheel angle in degrees as the disturbance input $w$; this is related to the steering angle $\delta$ by

$$
\delta=\frac{\pi}{180 \lambda} w
$$

where $\lambda$ is the steering ratio between the steering wheel and the wheels and is taken to be 18 .

For reasons discussed earlier, we choose $z_{1}=L T R_{d}$ given by (9) as one performance output; we want to keep $\left\|z_{1}\right\| \leq 1$ for the largest possible steering inputs. We consider the magnitude of the braking force $u$ to be limited by the weight $m g$ of the vehicle; so we choose $z_{2}=u$ as a second performance output. The resulting system with two performance outputs can be described by

$$
\begin{aligned}
\dot{x} & =A x+B w+B_{u} u \\
z_{1} & =C_{1} x \\
z_{2} & =u
\end{aligned}
$$

where

$$
B=\frac{\pi}{180 \lambda} B_{\delta}
$$

The parameters of the above model were tuned against the dynamics of a compact passenger vehicle such that there is a perfect match at steady state. The tuning was performed at $v=40 \mathrm{~m} / \mathrm{s}$ and with a step steering input of magnitude $30^{\circ}$. The corresponding tuned vehicle parameters are given in Table II.

First we obtain a control design which is based on the above model with a fixed speed; we call this the fixed model controller. We then consider the effect of varying speed in our control design and we obtain a control design assuming that the speed varies over some prespecified range; we call this the robust controller.

\section{A. Controller Based on Fixed Speed}

Here we base controller design on model (23) in which all matrices are constant and correspond to a fixed vehicle speed of $v=40 \mathrm{~m} / \mathrm{s}$. To obtain a state feedback controller, we applied Theorem 1. Since we desire that $\left\|z_{1}\right\| \leq 1$ and $\left\|z_{2}\right\| \leq m g$ for the largest possible steering inputs, we considered $\gamma_{2}=m g \gamma_{1}$. By performing a line search with respect to the scalar $\alpha$ we obtained a minimum value of 0.0089 for $\gamma_{1}$. The corresponding control gain matrix is

$$
K=m g \cdot\left[\begin{array}{llll}
-7.1287 & 0.9842 & 0.3271 & -0.0944
\end{array}\right] .
$$

Remark 2: Consider the constant speed model subject subject to the above control gain matrix. According to (21), the constraints on the outputs will not be violated for this constant speed closed loop system if the maximum magnitude $w_{\max }$ of the driver steering disturbance input satisfies $w_{\max } \leq 1 / \gamma_{1} \approx 112.97^{\circ}$. However application of the braking controller reduces vehicle speed. As the vehicle speed reduces, its tendency to rollover decreases and the vehicle can actually tolerate disturbances inputs with magnitude considerably larger than $1 / \gamma_{1}$. In simulations where the speed varies according to (5), the above controller gain matrix was able to maintain $\left|L T R_{d}\right| \leq 1$ and $\|u\| \leq m g$ for steering input magnitudes up to $w_{\max }=130^{\circ}$.

For numerical simulations we chose a driver steering input corresponding to an obstacle avoidance maneuver that is known as the elk-test; we chose an initial speed of $v=40 \mathrm{~m} / \mathrm{s}$ and a peak steering magnitude of $w_{\max }=130^{\circ}$. The steering profile corresponding to this maneuver and a comparison of speed histories
TABLE II

MODEL PARAMETERS

\begin{tabular}{cll}
\hline parameter & value & unit \\
\hline$m$ & 1224 & {$[\mathrm{~kg}]$} \\
$J_{x x}$ & 362.6 & {$\left[\mathrm{~kg} \cdot \mathrm{m}^{2}\right]$} \\
$J_{z z}$ & 1280 & {$\left[\mathrm{~kg} \cdot \mathrm{m}^{2}\right]$} \\
$l_{v}$ & 1.102 & {$[\mathrm{~m}]$} \\
$l_{h}$ & 1.25 & {$[\mathrm{~m}]$} \\
$T$ & 1.51 & {$[\mathrm{~m}]$} \\
$h$ & 0.375 & {$[\mathrm{~m}]$} \\
$c$ & 4000 & {$[\mathrm{~N} \cdot \mathrm{m} \cdot \mathrm{s} / \mathrm{rad}]$} \\
$k$ & 36075 & {$[\mathrm{~N} \cdot \mathrm{m} / \mathrm{rad}]$} \\
$C_{v}$ & 90240 & {$[\mathrm{~N} / \mathrm{rad}]$} \\
$C_{h}$ & 180000 & {$[\mathrm{~N} / \mathrm{rad}]$} \\
\hline
\end{tabular}
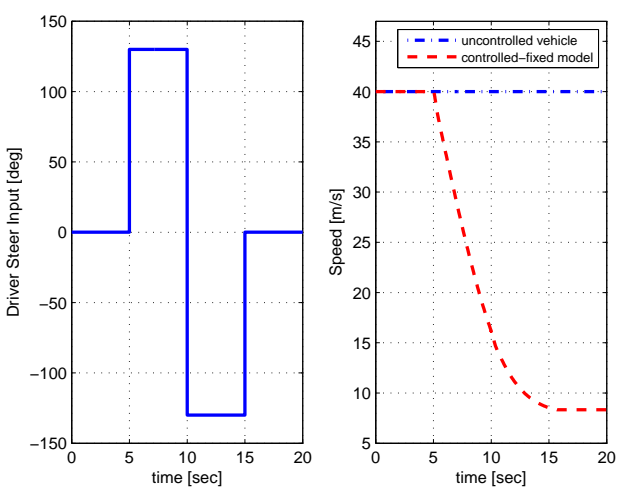

Fig. 3. Steering and speed histories.

for the controlled and uncontrolled vehicles are shown in Figure 3. Notice that, the dramatic speed drop of the controlled vehicle is a direct consequence of the braking action. In Figure 4 we further observe that $\left|L T R_{d}\right|>1$ for the uncontrolled vehicle throughout the manoeuver indicating possible rollover, whereas the vehicle with the proposed controller satisfies $\left|L T R_{d}\right|<1$ achieving the intended design goal and demonstrating the effectiveness of the proposed controller. Also for this maneuver, the peak value of the control force generated was about $80 \%$ of the total weight of the vehicle (i.e., $|u|<m g$ ), thus achieving the other design goal.

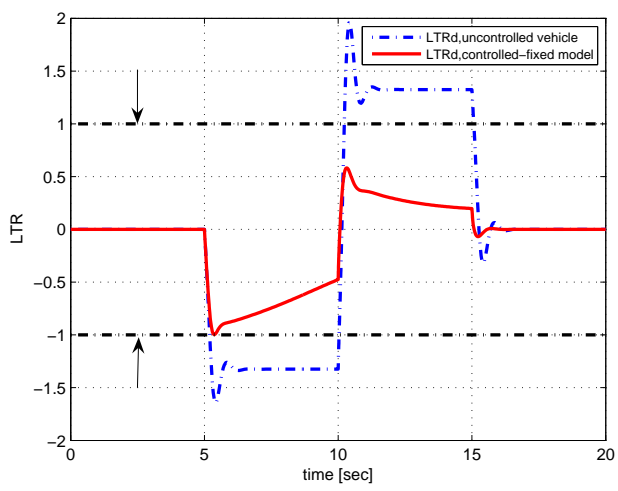

Fig. 4. Comparison of $L T R_{d}$ for the controlled and uncontrolled vehicles.

In the following subsection we demonstrate how our control design method can be extended to account for varying parameter uncertainties. 


\section{B. Controller Based on Variable Speed Model}

In this section, we present a rollover controller design which takes into account varying vehicle speed; it assumes constant model parameters given in Table II. We assume that the speed is bounded above and below by $\bar{v}$ and $\underline{v}$, respectively, that is, $\underline{v} \leq v \leq \bar{v}$. In order to represent typical freeway driving conditions for a compact passenger vehicle we chose $\underline{v}=25 \mathrm{~m} / \mathrm{s}$, and $\bar{v}=40 \mathrm{~m} / \mathrm{s}$ as the extremum design speeds. Again, we used the model (23) for controller design, where the matrices $A, B, B_{u}$ and $C_{1}$ are given in (4), (11) and (24). System matrices $B_{u}$ and $C_{1}$ are independent of speed. The matrices $A$ and $B$ can be expressed as affine linear functions of the time-varying parameters $\theta_{1}:=1 / v$ and $\theta_{2}:=1 / v^{2}$. These parameters are bounded as follows:

$$
\underline{\theta}_{1} \leq \theta_{1} \leq \bar{\theta}_{1}, \quad \underline{\theta}_{2} \leq \theta_{2} \leq \bar{\theta}_{2}
$$

where

$$
\underline{\theta}_{1}=\frac{1}{\bar{v}}, \quad \bar{\theta}_{1}=\frac{1}{\underline{v}}, \quad \underline{\theta}_{2}=\frac{1}{\bar{v}^{2}}, \quad \bar{\theta}_{2}=\frac{1}{\underline{v}^{2}} .
$$

Hence our system description satisfies Assumptions 1 and 2 with the following vertex matrices

$$
\begin{aligned}
& A_{1}=\bar{\theta}_{1} Y_{1}+\bar{\theta}_{2} Y_{2}+Y_{3}, \quad A_{2}=\bar{\theta}_{1} Y_{1}+\underline{\theta}_{2} Y_{2}+Y_{3}, \\
& A_{3}=\underline{\theta}_{1} Y_{1}+\bar{\theta}_{2} Y_{2}+Y_{3}, \quad A_{4}=\underline{\theta}_{1} Y_{1}+\underline{\theta}_{2} Y_{2}+Y_{3}, \\
& B_{1}=B_{2}=\left[\begin{array}{llll}
\frac{C_{v} J_{x e q}}{m J_{x x}} \bar{\theta}_{1} & \frac{C_{v} l_{v}}{J_{z z}} & \frac{h C_{v}}{J_{x x}} & 0
\end{array}\right]^{T}, \\
& B_{3}=B_{4}=\left[\begin{array}{llll}
\frac{C_{v} J_{x e q}}{m J_{x x}} \underline{\theta}_{1} & \frac{C_{v} l_{v}}{J_{z z}} & \frac{h C_{v}}{J_{x x}} & 0
\end{array}\right]^{T},
\end{aligned}
$$

where

$$
\begin{aligned}
Y_{1}= & {\left[\begin{array}{cccc}
-\frac{\sigma J_{x e q}}{m J_{x x}} & 0 & -\frac{h c}{J_{x x}} & \frac{h(m g h-k)}{J_{x x}} \\
0 & -\frac{\kappa}{J_{z z}} & 0 & 0 \\
0 & \frac{h \rho}{J_{x x}} & 0 & 0 \\
0 & 0 & 0 & 0
\end{array}\right], } \\
Y_{2}= & {\left[\begin{array}{cccc}
0 & \frac{\rho J_{x e q}}{m J_{x x}} & 0 & 0 \\
0 & 0 & 0 & 0 \\
0 & 0 & 0 & 0 \\
0 & 0 & 0 & 0
\end{array}\right], } \\
Y_{3}= & {\left[\begin{array}{cccc}
0 & -1 & 0 & 0 \\
\frac{\rho}{J_{z z}} & 0 & 0 & 0 \\
-\frac{h \sigma}{J_{x x}} & 0 & -\frac{c}{J_{x x}} & \frac{m g h-k}{J_{x x}} \\
0 & 0 & 1 & 0
\end{array}\right] . }
\end{aligned}
$$

We used Theorem 1 to design a controller which guarantees performance levels $\gamma_{1}$ and $\gamma_{2}=m g \gamma_{1}$, in presence of the any variations in speed satisfying $\underline{v} \leq v \leq \bar{v}$. We achieved $\gamma_{1}=0.009$. Also the corresponding control gain matrix is

$$
K=m g \cdot\left[\begin{array}{llll}
-7.5858 & 1.1995 & 0.3508 & -0.1478
\end{array}\right] .
$$

Note that, according to (21) the maximum theoretical driver steering disturbance input permitted is, $w_{\max }=1 / \gamma_{1} \approx 111.36^{\circ}$. In our simulations however, for the reasons explained in Remark 2, the robust controller was able to keep $\left|L T R_{d}\right| \leq 1$ for driver steering inputs with magnitudes up to $w_{\max }=136.5^{\circ}$.

For numerical simulations, we used the same obstacle avoidance (elk test) scenario as before, however with a peak driver steering input of magnitude $w_{\max }=136.5^{\circ}$ and an initial speed of $v=$ $40 \mathrm{~m} / \mathrm{s}$. The steering profile corresponding to this maneuver and a comparison of speed histories for the uncontrolled vehicle as well as the controlled vehicles with the two suggested control designs are shown in Figure 5. Notice here again that, the dramatic speed drop in the controlled vehicles is a direct consequence of the braking action. Also we observe that the speed loss due to the robust controller is slightly more than that due to the fixedmodel controller. Further results are presented in Figures 6 and 7, where we compare the performances of both the robust and the fixed-model controller designs. We observe in Figure 6 that, the $L T R_{d}$ due to the fixed-model controller slightly exceeds the lower boundary -1 at the initiation of the steering maneuver, while the robust controller results in $\left|L T R_{d}\right| \leq 1$ throughout the maneuver. In Figure 7 we compare the normalized control force histories for both of the controllers and observe that they are close and both result in $|u| \leq m g$ as desired.

It is of particular interest for us to see how the suggested controllers affect the vehicle path. To do this, we note that the coordinates $(x, y)$ of the vehicle CG relative to the road satisfy

$$
\begin{aligned}
& \dot{x}=v \cos (\beta+\psi), \\
& \dot{y}=v \sin (\beta+\psi),
\end{aligned}
$$

where we choose the initial coordinates $(x(0), y(0))$ to be zero. In Figure 8 the CG trajectories of the controlled and the uncontrolled vehicles are compared. Notice here that the shorter paths of the controlled vehicles are due to slowing down as a result of braking. We observe in Figure 8 that both controllers cause a small divergence from the intended vehicle path during the first half of the maneuver; in a real driving situation, the driver would time the second half of the maneuver based on the speed and location of the vehicle. Hence the second part of the maneuver would occur later for the controlled vehicles.
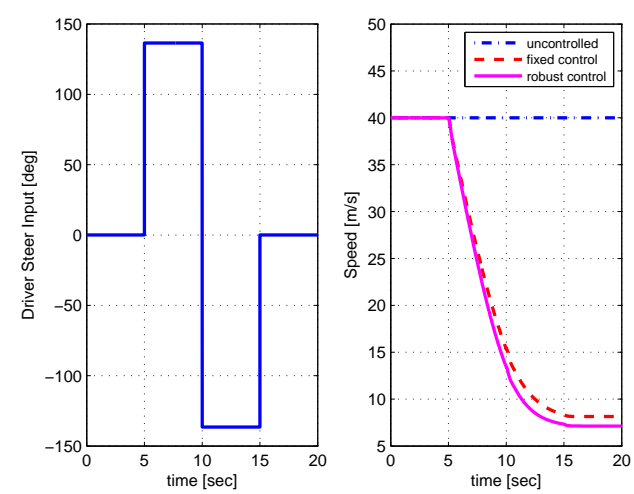

Fig. 5. Steering profile and a comparison of speed histories.

Comment : From the simulation results for the fixed model and the robust controllers, we observe that both controllers are effective in reducing the vehicle load transfer ratio $L T R_{d}$, and thus preventing rollover.

Comment : Our design is easily extended to incorporate other sources of parameter uncertainty such as the vehicle parameters, mass and center of gravity height.

\section{CONCLUSIONS}

We have presented a methodology for the design of vehicle rollover prevention systems using differential braking. By introducing the load transfer ratio $L T R_{d}$, we obtain a system performance output whose value provides an accurate measure for determining the onset of rollover. Our rollover prevention system is based upon recent results from Pancake, Corless and Brockman, which provide controllers to robustly guarantee that the peak values of the performance outputs of an uncertain system do not exceed certain 


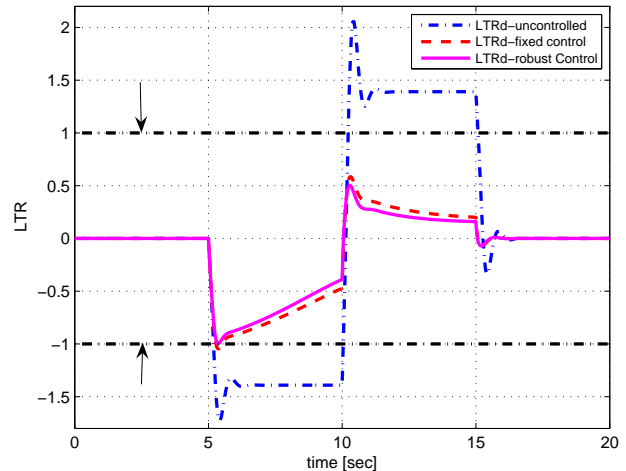

Fig. 6. Comparison of $L T R_{d}$ for the controlled and uncontrolled systems.

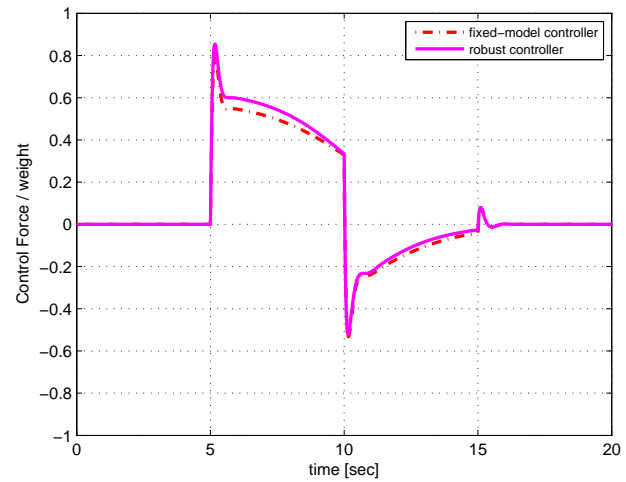

Fig. 7. Normalized control history comparisons.

values. Simulation results demonstrate the benefits of the proposed approach in a real-life problem.

Future work will proceed in several directions. We shall extend the methodology to include active steering, active suspension and combinations thereof to refine our rollover prevention strategy. Applications of control strategies with several actuators is not only limited to road and railroad vehicle roll stabilization, but can also be used to make the dynamics of a vehicle emulate those of another vehicle (e.g. having an SUV behave like a sports car). We shall also investigate a gain-scheduled version of our controllers and implement a switching strategy to improve their performance. Extension of our controller design for the case of tripped rollover is another future goal. Finally, we plan to implement and test our control system in real production vehicles with the help of our industrial partners.

\section{ACKNOWLEDGEMENTS}

This work was partially supported by Science Foundation Ireland Grant 04/IN1/I478.

\section{REFERENCES}

[1] National Highway Traffic Safety Administration (NHTSA), Traffic Safety Facts 2004: A Compilation of Motor Vehicle Crash Data from the Fatality Analysis Reporting System and the General Estimates System, Technical Report, 2006.

[2] Runge, J., Statement to the Committee on Commerce on SUV Safety, 2003, (available at http://www.nhtsa.dot.gov.)

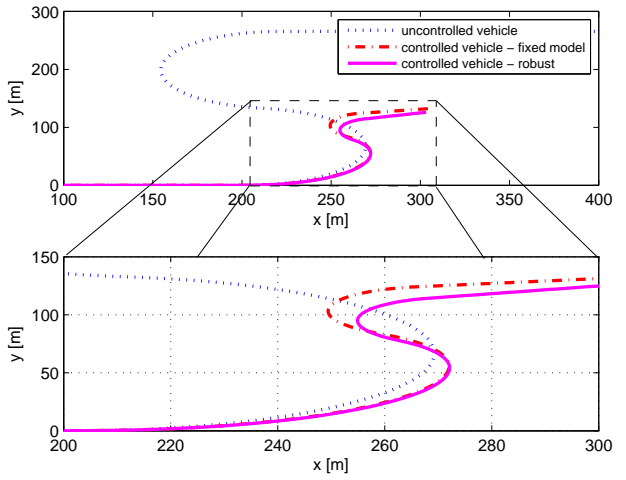

Fig. 8. Comparison of CG trajectories for the controlled and uncontrolled vehicle.

[3] Chou, C.C., McCoy, R.W. and Le, J., "A literature review of rollover test methodologies," Int. J. Vehicle Safety, Vol. 1, pp. 200-237, 2005.

[4] Chou, C.C., Wu, F., Gu, L. and Wu, S.R., "A review of mathematical models for rollover simulations," AMD-Vol. 230/BED-Vol. 41, Crashworthiness, Occupant Protection and Biomechanics in Transportation Systems, ASME, 1998.

[5] Carlson C.R. and Gerdes J.C., "Optimal rollover prevention with steer by wire and differential braking", Proceedings of the ASME International Mechanical Engineering Congress and Exposition, IMECE'03, Washington, D.C., USA, 2003.

[6] Pancake T., Corless M. and Brockman M., "Analysis and control of polytopic uncertain/nonlinear systems in the presence of bounded disturbance inputs", Proceedings of the American Control Conference, Chicago, IL, USA, 2000.

[7] Pancake T., Corless M. and Brockman M., "Analysis and Control of a class of uncertain/nonlinear systems in the presence of bounded disturbance inputs", in preparation.

[8] Palkovics L., Semsey À. and Gerum E., "Roll-over prevention system for commercial vehicles-additional sensorless function of the electronic brake system", Vehicle System Dynamics, Vol. 4, pp. 285-297, 1999.

[9] Wielenga T.J., "A method for reducing on-road rollovers: antirollover braking", SAE Paper No. 1999-01-0123, 1999.

[10] Chen B. and Peng H., "Differential-braking-based rollover prevention for sport utility vehicles with human-in-the-loop evaluations", Vehicle System Dynamics, Vol. 36, pp. 359-389, 2001.

[11] Ackermann J. and Odenthal D., "Robust steering control for active rollover avoidance of vehicles with elevated center of gravity", Proceedings of International Conference on Advances in Vehicle Control and Safety, Amiens, France, 1998.

[12] Odenthal D., Bünte T. and Ackermann J., "Nonlinear steering and braking control for vehicle rollover avoidance", Proceedings of European Control Conference, Karlsruhe, Germany, 1999.

[13] Kiencke U. and Nielsen L., Automotive Control Systems for Engine, Driveline and Vehicle, Springer-Verlag \& SAE Int., Berlin, 2000.

[14] Kamnik R., Böttiger F. and Hunt K., "Roll dynamics and lateral load transfer estimation in articulated heavy freight vehicles: A simulation study", Proceedings of the Institution of Mechanical Engineers, Part D, 2003.

[15] Akar M., Solmaz S. and Shorten R., "Method for determining the center of gravity for an automotive vehicle", 2006, patent pending.

[16] Solmaz S., Corless M. and Shorten R., "A methodology for the design of robust rollover prevention controllers for automotive vehicles: Part 2-Active steering", HYCON-CEMaCS Joint Workshop on Automotive Systems \& Control, June 1-2, 2006.

[17] Solmaz S., Corless M. and Shorten R., "A methodology for the design of robust rollover prevention controllers for automotive vehicles with active steering", under review. 